\title{
ANÁLISE ECONÔMICA DE REFLORESTAMENTO EM DOIS CICLOS DE PRODUÇÃO
}

Ana Paula da Silva Barros ${ }^{1}$ Luis Carlos de Freitas ${ }^{2}$ Matheus Macedo Sousa Lira ${ }^{3}$

${ }^{1}$ Acadêmica do curso de Engenharia Florestal da Universidade Estadual do Sudoeste da Bahia, apsbarros_eng3@hotmail.com

${ }^{2}$ Prof.Dr. do Depto de Fitotecnia e Zootecnia, da Universidade Estadual do Sudoeste da Bahia - UESB

${ }^{3}$ Engenheiro Florestal, Universidade Estadual do Sudoeste da Bahia.

Estrada do Bem-querer, $\mathrm{Km} 04$, Vitória da Conquista - BA 45.083-900 - Brasil

Recebido em: 03/10/2016 - Aprovado em: 21/11/2016 - Publicado em: 05/12/2016 DOI: 10.18677/EnciBio 2016B 156

\begin{abstract}
RESUMO
Os reflorestamentos de eucalipto têm expandido em várias regiões do país, todavia, tal crescimento não tem sido acompanhado sob a ótica da economia florestal. A falta de planejamento econômico na atividade pode, portanto, comprometer a sustentabilidade e a expansão do segmento florestal. Neste contexto, objetivou-se com este trabalho proceder um estudo econômico de empreendimentos florestais de eucalipto no estado da Bahia, com ênfase na produção madeireira, tomando como referência os seguintes indicadores: Valor Presente líquido (VPL), Razão Benefício/ Custo (RB/C); Custo Médio de Produção (CMP); Valor Periódico equivalente (VPE) e Taxa Interna de Retorno (TIR). Após determinação dos indicadores econômicos, projetou-se um novo cenário com alterações na taxa mínima de atratividade (TMA); valor de mercado da madeira, custo de implantação florestal e incremento médio anual. Os cinco indicadores avaliados mostraram viabilidade para a taxa mínima de atratividade adotada (6\% a.a). Na sensibilidade, apesar da retração em alguns parâmetros, todos os cenários mostraram atrativos.
\end{abstract}

PALAVRAS CHAVE: Economia florestal, projeto florestal, reflorestamento.

\section{ECONOMIC ANALYSIS OF REFORESTATION IN TWO PRODUCTION CYCLES}

\begin{abstract}
The forest enterprises have expanded in several regions of the country, however, most of the time, growth is not accompanied from the perspective of forestry. The lack of economic planning can undermine the sustainability and expansion of the forestry activity. The objective of this study was to carry out an economic study of forest enterprises of eucalyptus in the state of Bahia, using Present Net Value (VPL) value equivalent Periodic Review (VPE), Internal Rate of Return (TIR), Benefit Cost Ratio (RB /C) Production and Average Cost (CMP). After determining the economic indicators proceeded to a sensitivity study regarding interest rates, selling price of


wood, cost of reforestation and average annual increment. The five indicators presented economically feasible for an interest rate of $6 \%$ a.a. In the Sensitivity, despite shrinkage in some parameters, all scenarios showed attractive.

KEYWORDS: Forest economics, forest project, reforestation.

\section{INTRODUÇÃO}

O setor florestal brasileiro é responsável por uma parcela do desenvolvimento econômico e sustentável do país, contextualizando um modelo com plenas condições de oferecer melhor distribuição de renda e condições dignas de sobrevivência para a população. Neste contexto, surge a economia que estuda a forma como as pessoas e a sociedade decidem empregar recursos de produção escassos e que poderiam ter utilização alternativa na produção de bens e serviços de forma a distribuí-los entre as várias pessoas e a sociedade, a fim de satisfazer as necessidades humanas (SILVA \& SOARES, 2009). Avaliando sob a ótica da economia florestal, tais recursos poderiam ser entendidos como a terra, mão de obra e maquinários, os quais irão contextualizar, de forma conjunta, um cenário de produtividade para o segmento em questão.

Em relação a projeção do setor florestal, vem se verificando o aumento anual da produção, principalmente dos gêneros Eucalyptus e Pinus, totalizando no ano de 2013 uma área de 7,2 milhões de hectares (IBA, 2014). Mais especificamente no estado da Bahia, os reflorestamentos apresentaram um crescimento de $6,3 \%$ em comparação com o ano de 2013 (ABAF, 2015). Provavelmente, grande parte dessa ampliação se deve a elasticidade do eucalipto em relação a variedade de multiprodutos, o que torna o produto final extremamente abrangente, possuindo vários segmentos como papel e celulose, siderurgia, painéis reconstituídos, produtos farmacológicos, dentre outros (ALVES et al., 2015).

Apesar da diversificação para o uso da matéria-prima de eucalipto e sua maior facilidade de aceitação no mercado, empreendimentos dessa natureza caracterizam-se por ser um investimento de longa duração, exigindo consciência e diligência na política e planejamento, mas, sobretudo, nas práticas de gestão, a fim de evitar impactos negativos (ABRAF, 2013). Por se tratarem de investimentos de grandes horizontes, tais empreendimentos requerem, quando do processo de gestão financeira, que os custos sejam apurados e devidamente descontados para uma mesma base, podendo ser para o presente, através do valor presente, ou para o futuro, através do valor futuro (GRAÇA et al. 2000). Da mesma forma, se faz necessário também descontar as receitas para uma mesma base.

A economia florestal permite ao administrador tomar decisões mais seguras e lucrativas, garantindo o sucesso do empreendimento (SILVA et al. 2005) norteando ainda limites seguros de investimentos, principalmente quando das projeções dos indicadores relacionados as alterações nas taxas de juros, preço de mercado da madeira, ou variações no custo de produção.

Diante do crescimento que o segmento tem alcançado na Bahia e da escassez de pesquisas que avaliem economicamente projetos florestais no estado, objetivou-se com esta pesquisa realizar um estudo econômico e de sensibilidade em projetos florestais de eucalipto localizados no litoral norte da Bahia. 


\section{MATERIAL E MÉTODOS}

\section{Área de estudo}

O estudo foi realizado no município de Pojuca, Bahia, pertencente à região metropolitana de Salvador. Segundo classificação de Köppen (SILVA, 2005) o clima é do tipo Af, quente e úmido e, chuvoso, com precipitações durante o ano entre 1300 a $2300 \mathrm{~mm}$, sem estação seca.

\section{Indicadores econômicos analisados}

Elaborou-se um fluxo de caixa incluindo as atividades de implantação, condução e colheita florestal (Tabela 1). Os indicadores avaliados encontram-se no Quadro 1. Segundo REZENDE \& OLIVEIRA (2013) esses métodos consideram o valor do capital no tempo, ou seja, atribuem diferentes ponderações às receitas líquidas em função da distribuição ao longo do tempo, sendo os mais indicados para a análise de projetos no setor florestal.

QUADRO 1 - Parâmetros utilizados para análise da viabilidade econômica.

\begin{tabular}{|cc|}
\hline VPL & $V P L=\sum_{j=0}^{n} R j(1+i)^{-j}-\sum_{j=0}^{n} C j(1+i)^{-j}$ \\
\hline VPE & $V P E=\frac{V P L\left[(1+i)^{-t}-1\right]}{\left[1-(1+i)^{-n t}\right]}$ \\
\hline TIR & $\sum_{j=0}^{n} R_{j(1+T I R)^{-j}}=\sum_{j=0}^{n} C_{j(1+T I R)^{-j}}$ \\
\hline B/C & $B / C=\frac{\sum_{i=0}^{n} R_{j(1+i)^{-j}}}{\sum_{i=0}^{n} C_{j(1+i)^{-j}}}$ \\
\hline CMP & $C M P=\frac{\sum_{j=0}^{n} C T j}{\sum_{j=0}^{n} Q T j}$ \\
\hline
\end{tabular}

Onde: $\mathrm{i}=$ taxa juros (a.a); $\mathrm{t}=$ número de períodos de capitalização. $\mathrm{Rj}=$ valor atual das receitas; $\mathrm{C}$ = valor atual dos custos; $\mathrm{j}=$ período em que a receita ou custo ocorrem; $\mathrm{n}=$ duração do projeto em anos; $\mathrm{CTj}=$ custo total equivalente; $\mathrm{QTj}$ = produção total equivalente; TIR = taxa interna de retorno.

\section{Estudo da sensibilidade}

Realizou-se algumas projeções contextualizando possíveis alterações em relação a taxa de juros, preço de mercado da madeira, custos de implantação florestal (Tabela 3) e incremento médio anual (Figura 2). 


\section{RESULTADOS E DISCUSSÃO}

As análises foram realizadas com base em um fluxo de caixa do empreendimento (Tabela 1). Os custos e receitas ocorridos no mesmo ano foram processados (receitas - custos), condicionando um balanço econômico ao longo de todo horizonte de planejamento do empreendimento. Os custos englobaram os seguintes processos: implantação, condução, arrendamento da terra e colheita florestal (Tabela 1). Considerando apenas os custos mais expressivos ocorridos na implantação e no primeiro ano, observou-se um aumento de $25,90 \%$ em relação aos valores encontrados por CARMO et al. (2011). Apesar da diferença, percebe-se a equivalência dos valores se considerar uma taxa de juros de $6,00 \%$ a.a. corrigida entre os anos de 2011 e 2015 (ano de realização da presente pesquisa).

As receitas foram auferidas mediante 0 produto dos fatores produtividade (IMA=39 $\left.\mathrm{m}^{3} / \mathrm{ha} / \mathrm{ano}\right)$, idade de corte $\left(6\right.$ anos) e preço da madeira ( $\left.R \$ 45,00 / \mathrm{m}^{3}\right)$, conforme cotação realizada no ano de 2015.

TABELA 1- Fluxo de Caixa para o empreendimento florestal avaliado.

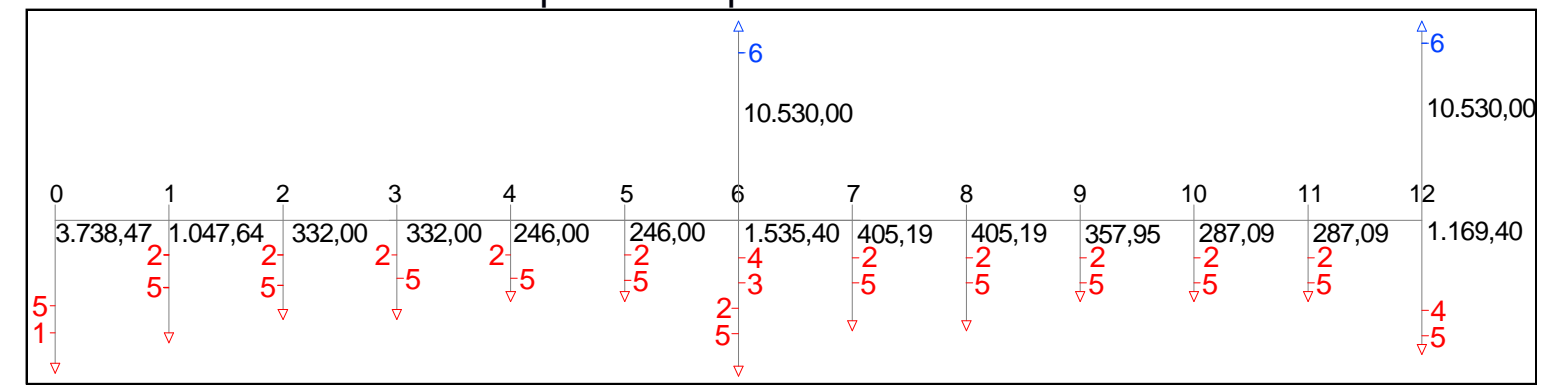

Onde: 1=Custos de Implantação; 2=Custos de tratos culturais; 3=Condução de brotação; 4=Custos da colheita; $5=$ Custo de arrendamento da terra; $6=$ Volumes $1^{\circ}$ e $2^{\circ}$ corte $\left(234 \mathrm{~m}^{3} / \mathrm{ha}\right)$.

\section{Análise Econômica}

Verificou-se que o empreendimento avaliado mostrou-se atrativo para todos os parâmetros analisados. O VPL indicou que foi possível obter uma renda líquida de $R \$ 4.265,94$ por hectare de floresta, considerando um horizonte de 12 anos. Embora o referido método não seja adequado para comparar projetos com horizontes diferentes, é um dos métodos que normalmente apresenta resultado correto (SILVA \& SOARES, 2009).

\begin{tabular}{|c|c|}
\hline $\begin{array}{l}\text { Indicadores } \\
\text { Econômicos }\end{array}$ & $\begin{array}{l}\text { Resultados } \\
\text { da Análise }\end{array}$ \\
\hline VPL & $\mathrm{R} \$ 4.265,94 / \mathrm{ha}$ \\
\hline VPE & $\mathrm{R} \$ 508,83$ \\
\hline $\begin{array}{c}\text { TIR } \\
\mathrm{RB} / \mathrm{C} \\
\mathrm{CMP}\end{array}$ & $\begin{array}{c}14,20 \% \\
1,51 \\
\mathrm{R} \$ 29,83 / \mathrm{m}^{3}\end{array}$ \\
\hline
\end{tabular}

O VPE evidenciou que o projeto tem capacidade de gerar receitas anuais na ordem de $\mathrm{R} \$ 508,83$ tornando-o, portanto, viável (VPE>0). A grande vantagem desse 
indicador na análise de viabilidade econômica de projetos está no fato de corrigir, implicitamente, as diferenças de horizonte (REZENDE \& OLIVEIRA, 2013).

A Taxa Interna de Retorno foi determinada através do método matemático. Segundo SILVA et al. (2005), a determinação desse índice normalmente é efetuada por meio de programas computacionais que fazem os cálculos até encontrar a taxa que anula o VPL. O valor obtido para a TIR foi de $14 \%$, o que fez com que 0 empreendimento avaliado apresentasse viável também por esse método, uma vez que a TIR foi superior a taxa mínima de atratividade (6,00\%). O índice encontrado para a Razão Benefício Custo $(\mathrm{B} / \mathrm{C})$ indicou que o quantitativo de receitas superou em $51 \%$ os custos do projeto.

O Custo Médio de Produção encontrado, considerando o ano de 2015, foi de $\mathrm{R} \$ 29,83 / \mathrm{m}^{3}$ sendo o preço de mercado da madeira cotado em $\mathrm{R} \$ 45,00$. Conclui-se, portanto, que houve um ganho real de $\mathrm{R} \$ 15,17$ para cada metro cúbico de madeira comercializado. VIRGENS et al., (2016), realizando estudo da mesma natureza, observaram, em relação ao indicador CMP, um lucro na ordem $R \$ 12,32$.

\section{Análise de Sensibilidade}

A referida análise não mostrou condições de inviabilidade para 0 empreendimento avaliado, apesar da retração de alguns indicadores (Tabela 3). SILVA (2014), avaliando a sensibilidade de um empreendimento florestal de eucalipto, observou que o projeto foi viável para taxas de juros de até $7,54 \%$ a.a., volume mínimo de colheita de $213 \mathrm{~m}^{3} /$ hectare ao $7^{\circ}$ ano, e preço mínimo de $R \$$ $38,00 / \mathrm{m}^{3}$ para madeira empilhada (mercado de torete entre 15 a $30 \mathrm{~cm}$ ).

TABELA - 3. Análise de sensibilidade considerando taxa de juros, produção e preço da madeira.

\begin{tabular}{cccccc}
\hline \multicolumn{6}{c}{ Análise Econômica } \\
\hline Taxa de Juros & VPL (R\$) & TIR & VPE (R $\$ /$ ha/ano) & B/C & CMP $\left(\mathrm{R} \$ / \mathrm{m}^{3}\right)$ \\
\hline $6 \%$ & $4.265,94$ & - & 508,83 & 1,51 & 29,83 \\
$8 \%$ & $2.889,90$ & - & 383,48 & 1,36 & 34,90 \\
$10 \%$ & $1.764,27$ & - & 258,93 & 1,23 & 46,98 \\
\hline \multicolumn{5}{c}{ Preço da Madeira $(\mathrm{R} \$ 45,00)$} \\
Redução de 10\% & $3.132,99$ & $12 \%$ & 373,69 \\
Padrão & $4.265,94$ & $14 \%$ & 508,83 & 1,36 & - \\
Adicional de 10\% & $5.531,57$ & $16 \%$ & 659,79 & 1,66 & - \\
\hline Redução de 15\% & $4.826,71$ & $16 \%$ & 575,72 & 1,62 & 27,84 \\
Padrão & $4.265,94$ & $14 \%$ & 508,83 & 1,51 & 29,83 \\
Adicional de 15\% & $3.705,17$ & $13 \%$ & 441,94 & 1,41 & 31,83 \\
\hline
\end{tabular}

Aumentos de $2 \%$ e $4 \%$ na taxa de juros proporcionaram reduções nos valores de VPL na ordem de $R \$ 1.376,04$ (32,26\%) e $R \$ 2.501,67$ (58,64\%), respectivamente (Tabela 4). VITALE \& MIRANDA (2010) confirmam esta tendência de retração para o respectivo indicador, quando do aumento da taxa mínima de 
atratividade. Redução de $10 \%$ no preço de mercado da matéria-prima (madeira) proporcionou retração no fluxo anual de receitas (VPE) na ordem de $R \$ 1.132,95$ (Tabela 3). Estudos realizados por ALVES et al. (2015) evidenciaram, como variáveis mais influentes nos valores do VPL, VPE e da TIR, o preço de venda da madeira $\left(m^{3} / h a\right)$, produção $\left(m^{3} / h a\right)$ e preço da terra $(R \$ / h a)$.

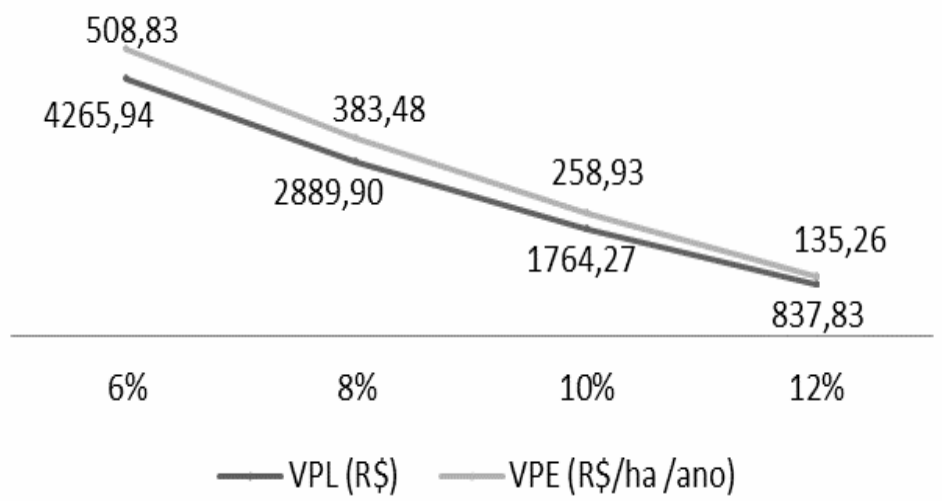

FIGURA 1 - Análise de sensibilidade para os critérios VPL e VPE em função das taxas de juros.

Os resultados mostraram que a tendência de viabilidade, segundo os critérios avaliados, foi inversamente proporcional ao aumento da taxa anual de juros, com exceção do CMP. O VPL e o VPE apresentaram o mesmo comportamento, porém, o VPL mostrou-se mais sensível a variação na taxa de juros (Figura 1). Considerando oscilações no Incremento Médio Anual, os indicadores VPL e VPE apresentaram valores proporcionais (Figura 2).

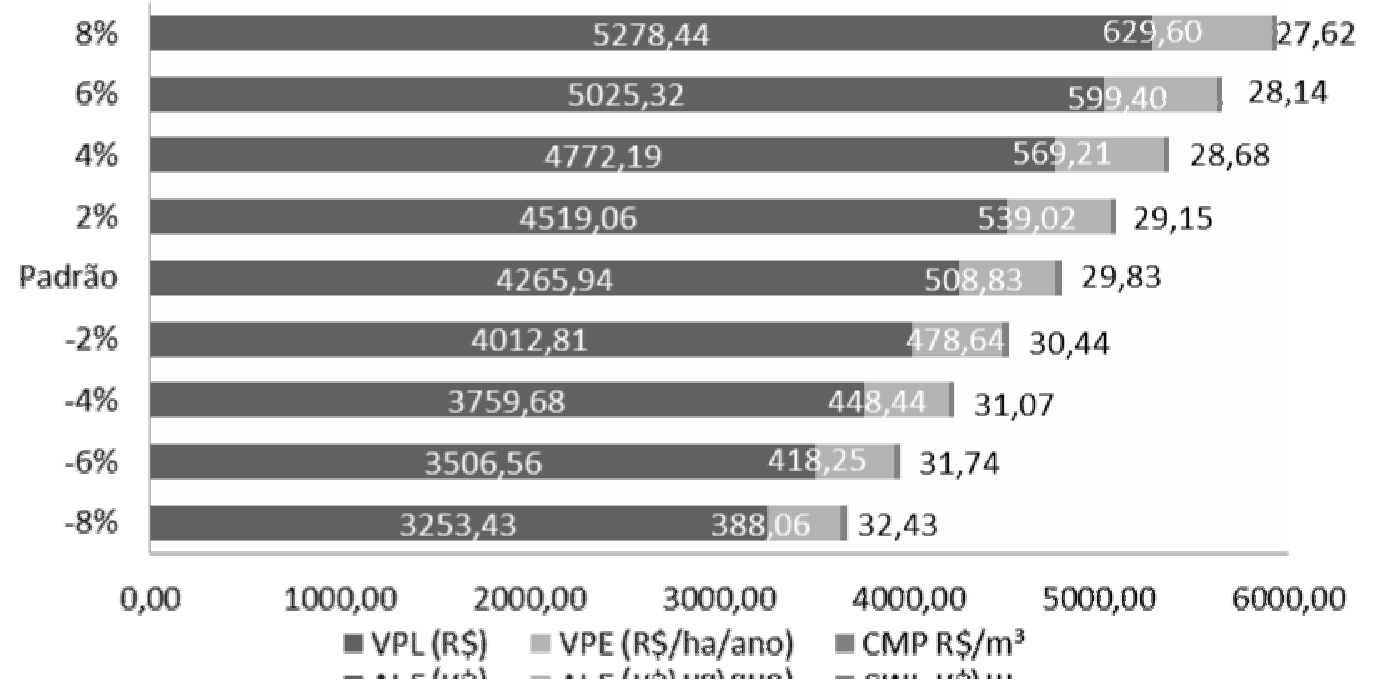

FIGURA 2 - Análise de sensibilidade para os critérios VPL, VPE e CMP em função do incremento médio anual. 


\section{CONCLUSÃO}

O empreendimento em questão mostrou-se viável economicamente para todos os indicadores avaliados. O estudo de sensibilidade evidenciou riscos econômicos para uma possível flexibilização na taxa de juros, preço da madeira e nos custos de implantação florestal.

\section{REFERÊNCIAS}

ABRAF. Anuário estatístico ABRAF 2013, ano base 2012 / ABRAF. - Brasília: 2013.

ASSOCIAÇÃO BAIANA DAS EMPRESAS DE BASE FLORESTAL - ABAF. Bahia Florestal Anuário da ABAF 2015: ano base 2014. Bahia: ABAF; 2015.

ALVES, T. R; CORDEIRO, S. A; OLIVEIRA, M.L.R; LACERDA, K. W. S. MENDES, R. $\mathrm{T}$. Influência do custo da terra na viabilidade econômica de plantios de eucalipto no Vale do Jequitinhonha-MG. Reflexões Econômicas. v. 1, n. 1. 2015, p. 131-151.

CARMO, F. C. A.; FIEDLER, N. C.; GUIMARÃES, P. P.; PEREIRA, D. P.; ANDRADE, W. S. P. Análise de custos da implantação de cultivos de eucalipto em áreas acidentadas no sul do Espírito Santo. Cerne, Lavras, v. 17, n. 4, p. 473-479, 2011.

GRAÇA, L. R.; RODIGHERI, H. R.; CONTO, A. J.; Custos florestais de produção: conceituação e aplicação. Colombo: Embrapa Florestas, 2000. 32p. (Embrapa Florestas. Documentos, 50). Disponível em: http://www.cnpf.embrapa.br/publica/seriedoc/edicoes/doc50.pdf.

INDÚSTRIA BRASILEIRA DE ÁRVORES (IBA). O setor em números, 2014. 21p. Disponível em: http://www.bracelpa.org.br/shared/2014.07.02.booklet-pt.pdf.

REZENDE, J. L. P.; OLIVEIRA, A.D.; Análise econômica e social de projetos florestais. 3 ed. Ver. Ampl. Viçosa, MG: Ed. UFV, 2013. 385p.

SILVA, J.O. Análise econômica de projeto de silvicultura com eucalipto (Eucalyptus spp) utilizando programação linear: estudo de caso de uma propriedade rural do Distrito Federal - DF. Trabalho de conclusão de curso de especialização em gestão florestal. 2014. 35 p.

SILVA, M. L.; JACOVINE, L. A. G.; VALVERDE, S. R. Economia Florestal. 2 ED. Viçosa, MG: UFV, 2005. 178 P.

SILVA, M.L.; SOARES, N.S. Exercícios de economia florestal: aprenda praticando. Viçosa, MG. 2009. p141 p.

VIRGENS, A. P.; FREITAS, L.C.; LEITE, A.M.P. Análise Econômica e de Sensibilidade em um Povoamento Implantado no Sudoeste da Bahia. Revista Floresta e Ambiente. Seropédica-RJ, vol.23, n.2, p. 211-219, 2016. Disponível em: DOI: $10.1590 / 2179-8087.104914$. 
VITALE, V; MIRANDA, G.M. Análise comparativa da viabilidade econômica de plantios de Pinus taeda e Eucalyptus dunnii na região centro Sul do Paraná. Revista Floresta. Curitiba, PR, v. 40, n. 3, p. 469-476. 2010. 